\title{
DEM Analysis of Particle Adhesion during Powder Mixing for Dry Powder Inhaler Formulation Development
}

\author{
Jiecheng Yang ${ }^{1, *}$ Chuan-Yu Wu ${ }^{2, \dagger}$, Michael Adams ${ }^{1}$ \\ ${ }^{1}$ School of Chemical Engineering, University of Birmingham, B15 2TT, UK \\ ${ }^{2}$ Department of Chemical and Process Engineering, University of Surrey, \\ Guildford, GU2 7XH, UK
}

\begin{abstract}
Understanding the adhesive interactions between active pharmaceutical ingredient (API) particles and carrier particles in dry powder inhalers (DPIs) is critical for the development of formulations and process design. In the current study, a discrete element method (DEM), which accounts for particle adhesion, is employed to investigate the attachment processes in DPIs. A critical velocity criterion is proposed to determine the lowest impact velocity at which two elastic autoadhesive spherical particles will rebound from each other during impact. Furthermore, the process of fine API particles adhering to a large carrier in a vibrating container is investigated. It was found that there is an optimal amplitude and frequency for the vibration velocity that can maximise the number of particles contacting with the carrier (i.e. the contact number). The impact number and detachment number during the vibration process both increase with increasing vibration amplitude and frequency while the sticking efficiency decreases as the amplitude and frequency is increased.
\end{abstract}

Key words: adhesion; discrete element method; attachment; mixing; granular materials

\footnotetext{
* Corresponding author. Tel: +44-01214145365. Fax: +44-01214145324 Email: jxy147@bham.ac.uk

Previously with School of Chemical Engineering, University of Birmingham, B15 2TT, UK.
} 


\section{Introduction}

Dry powder inhalers (DPIs) are widely used for treating pulmonary and respiratory diseases, which can directly deliver micron-sized active pharmaceutical ingredients (API) particles to the lungs and respiratory tracts [1-3]. Since the API particles are generally very small $(<5 \mu \mathrm{m})$, they are extremely cohesive and have poor flowability [4]. To improve the flowability and dispersion efficiency of DPI formulations, API particles are either mixed with large carrier particles (usually $30-70 \mu \mathrm{m}$ ) or aggregated into large agglomerates [5]. The attractive potential between API and carrier particles or other API particles is mainly due to van der Waals forces, electrostatic forces and capillary forces [6]. The particle-particle interaction forces have to be sufficiently strong that fine API particles can adhere with the carriers or form large agglomerates to be transported, but weak enough that they can be detached or dispersed by a deagglomeration process and thus allow the delivery of the API particles into the respiratory tracts and lungs. Therefore, particle-particle interactions play a significant role in controlling the performance of DPIs and hence many studies have been performed to explore the dependency of these interactions on particle size $[7,8]$, material properties $[9,10]$, particle concentration [11,12], particle morphology [13,14], particle surface roughness [15,16], storage conditions [17,18], surface area [19], density and porosity [20], and crystal form [21]. For example, Kaialy et al. [7] experimentally examined the influence of the particle size of lactose carrier on the DPI performance and demonstrated that the DPI performance improved with decreasing carrier particle size. Young et al. [11] investigated the relationship between API dosage (drug/lactose ratio) and aerosolisation performance of conventional carrier based formulations using the twin stage impinger and found that the fine particle fraction (FPF) decreased with increasing API dosage for small values of the parameter $(10-135 \mu \mathrm{g} / 50 \mathrm{mg}$ ), while the FPF increased with increasing API dosage at large values of the API dosage $(135-450 \mu \mathrm{g} / 50 \mathrm{mg})$. These results were attributed to the effect of "active sites", which were preferentially occupied by API particles due to large contact area, high 
surface energy and simple geometric constrains. Therefore, there was a critical API dosage below which FPF decreased since API particles were adhered to active sites, and above which FPF increased since active sites were fully occupied with excessive API particles present in the formulation. Kaialy et al. [15] also found that lactose particles with an elongated shape, more irregular shapes and a rougher surface could delivery more API particles to lower airway regions. Le et al. [17] employed a twin stage impinger to evaluate the dispersion performance at various humidity conditions and found that the FPF decreased as the relative humidity is increased. Cline and Dalby [19] found that the FPF increased with the increased surface area. Kaialy and Nokhodchi [20] suggested that engineered lactose with smaller bulk and tap density and higher porosity improved the homogeneity of drug content and the dry powder inhaler performance. Shur et al. [21] used seed crystals and supercritical carbon dioxide crystallization to produce ipratropium bromide monohydrate and anhydrous crystals, respectively, and found that these two crystals exhibited similar mechanical and interfacial properties, which also results in the similar DPI performance.

As the first step in preparing DPI formulations, the attachment of API particles to carrier particles is a critical stage for controlling the performance of DPIs. This is generally realised through mixing APIs with carriers in a blender, which determines the formulation quality and efficiency [17,22-24]. Both mixing conditions (e.g. mixing time, mixing speed) and mixing processes can influence the formulation behaviour. For example, Selvam et al. [22] explored the effect of mixing time on the drug attachment process and found that the drug loading increased with increasing mixing time. They argued that this was due to the formation of drug-drug aggregates on the surface of the carrier particles as more drug particles were adhered over time. However, Le et al. [17] investigated the influence of mixing conditions on the API content and demonstrated that the mean values of the blends were poorly correlated with the mixing time. These discrepancies might be caused by the different mixing times used (i.e. 1-30 min by Selvam et al. [22] and 60-180 min by Le et al. [17]) and different materials investigated (i.e. Selvam et al. [22] used budesonide 
drug and polyolefin medical packaging films, while Le et al. [17] considered lactose carrier and Fluticasone Propionate drug).

The effect of mixing processes on formulation properties and DPI performance has also been investigated [23-26]. Johns et al. [23] showed that the addition of fine excipient particles during the mixing process increased the pulmonary delivery of the drug and there was an optimal concentration of added excipients . Zeng et al. [24] used a twin stage impinger to investigate the effect of different sequences of addition on in vitro deposition. They found that the formulation prepared by first blending micronized lactose with coarse lactose before mixing with salbutamol sulphate produced a greater fine particle fraction and fine particle dose of salbutamol sulphate than the same formulation prepared using a different mixing order of the three components. Saleem et al. [25] used two lactose carrier systems (spray-dried and milled) and investigated the effects of surface energy and the so-called 'rate of blending' on the aerosol dispersion performance. They observed that the surface energy was inversely proportional to the DPI performance while the blending rates positively correlated with the performance. Dickhoff et al. [26] explored the effect of carrier payload $(0.4-6.0 \% \mathrm{w} / \mathrm{w}$ of $\mathrm{drug})$ on the dispersion performance of drug particles from adhesive mixtures and demonstrated that the amount of the residual drug on the carrier decreased with increasing carrier payload at a flow rate of $60 \mathrm{l} / \mathrm{min}$. All these studies demonstrated that mixing is crucial in controlling the formulation quality, efficiency and performance of DPIs. Therefore, an improved understanding of the mechanism of particle adhesion and the drug attachment process during mixing is critical for enhancing not only the formulation quality, but also the performance of DPIs.

Although many efforts have been made to investigate the attachment process of DPI formulations, the underlying mechanisms, especially at the microscopic level, are still not well understood. Therefore, a Discrete Element Method (DEM) analysis is reported in this paper in order to provide insights into the attachment process during mixing. The impact between two spheres is first analysed to establish a criterion for determining whether two particles will attach or rebound during an impact. 
In addition, the adhesive interaction between API particles and a large carrier particle in a vibrating container is analysed and the effects of vibration conditions are examined.

\section{DEM with adhesion}

The DEM for adhesive particles developed by Thornton and Yin [27] is used in this study. The translational and rotational motions of a particle are governed by the Newton's second law:

$$
\begin{gathered}
m_{i} \frac{\mathrm{d} \mathbf{v}_{i}}{\mathrm{~d} t}=\mathbf{f}_{c i}+m_{i} \mathbf{g} \\
I_{i} \frac{\mathrm{d} \boldsymbol{\omega}_{i}}{\mathrm{~d} t}=\mathbf{T}_{i}
\end{gathered}
$$

where $m_{i}, I_{i}, \mathbf{v}_{i}, \boldsymbol{\omega}_{i}$ are the mass, moment of inertia, translational and rotational velocities of particle $i$, respectively. $\mathbf{f}_{c i}$ and $\mathbf{T}_{i}$ are the contact force and torque acting on the particle. $\mathbf{g}$ is the gravitational acceleration. Due to the small particle sizes of both APIs and carriers used in DPIs, adhesion becomes important in governing the interaction between particles and particles/walls. Therefore, JKR theory [28] is applied to model the adhesion between particles, in which the relationship between the normal contact force $P$ (i.e. normal component of $\mathbf{f}_{c i}$ ) and relative normal displacement $\alpha$ is given as [29]:

$$
\frac{\alpha}{\alpha_{F}}=\frac{3\left(\frac{P}{P_{C}}\right)+2 \pm 2\left(1+\frac{P}{P_{C}}\right)^{1 / 2}}{3^{2 / 3}\left[\frac{P}{P_{C}}+2 \pm 2\left(1+\frac{P}{P_{C}}\right)^{1 / 2}\right]^{1 / 3}}
$$

where $\alpha_{F}$ and $P_{C}$ are the displacement at which the two adhesive spheres are separated and the "pull-off" force with which the two adhesive spheres can be separated, and given as:

$$
\alpha_{F}=\frac{3}{4}\left(\frac{\pi^{2} \Gamma^{2} R}{E^{* 2}}\right)^{1 / 3}=\left(\frac{3 P_{C}{ }^{2}}{16 R E^{* 2}}\right)^{1 / 3}
$$




$$
\begin{array}{r}
P_{C}=\frac{3}{2} \Gamma \pi R \\
R=\frac{R_{1} R_{2}}{R_{1}+R_{2}} \\
\frac{1}{E^{*}}=\frac{1-v_{1}^{2}}{E_{1}}+\frac{1-v_{2}{ }^{2}}{E_{2}}
\end{array}
$$

in which $\Gamma$ is the thermodynamic work of adhesion, and $R$ is the radius, $v$ is the Poisson's ratio, and $E$ is the Young's modulus of the particles 1 and 2.

\section{DEM models}

Using DEM, impacts between two adhesive particles and mixing of API particles with a carrier particle in a vibrating container are simulated. The corresponding DEM models are presented in this section.

\subsection{Impact between two spheres}

Two identical particles with the properties given in Table 1 are created to impact collinearly with each other in the presence of adhesion as shown in Fig. 1. The initial velocities of the two particles are $V_{1}$ and $V_{2}$, which have

the same magnitudes but are in opposite directions (i.e. $V_{1}=-V_{2}$ ). Gravity is not considered in this case.

\subsection{Mixing of API particles with a carrier particle in a vibrating container}

Mixing of one carrier particle and $N$ API particles in a vibrating cubic container is modelled in 3D. The side length of the container is $l$. All the carrier and API particles are spherical particles. The diameter of the carrier particle and mono-sized API particles are $70 \mu \mathrm{m}$ and $5 \mu \mathrm{m}$, respectively. The carrier particle is initially located at the centre of the container while the API particles are randomly generated in the empty space in the container as shown in Fig. 2a. The carrier and API particles are then allowed to settle to the bottom of the container under gravity as shown in Fig. 2b. Once the kinetic energy of the system (carrier and API particles) 
becomes negligible, the container is set to vibrate vertically with a velocity profile defined as:

$$
\begin{gathered}
V_{y}=V_{0} \sin (2 \pi f t) \\
V_{x}=V_{z}=0
\end{gathered}
$$

where $V_{x}, V_{y}$ and $V_{z}$ are the velocity components of the container, $V_{0}$ is the amplitude, $f$ is the frequency, and $t$ is the time. The simulation parameters are given in Table 2. It is assumed that the API particles and the carrier have the same material properties as a-lactose monohydrate [30].

\section{Impact between two particles with adhesion}

\subsection{Impact behaviour}

Fig. 3 shows the evolution of the relative displacement, relative velocity and normal contact force during the impact of two spheres at $V_{1}=-V_{2}$ $=0.0058 \mathrm{~m} / \mathrm{s}$. The abscissa shows the time normalized by the duration from the beginning of the contact until the time instant at which the relative displacement reduces to zero. It can be seen that for autoadhesive particles, the relative displacement initially increases until a maximum value is reached, when the relative velocity reaches zero. Thereafter the relative displacement reduces. However, due to the adhesion, the two spheres cannot separate from each other when the relative displacement reduces to zero, and they continue to move in opposite directions until the relative displacement reduces to a certain value (i.e. $\alpha_{F}$ as described in Eq. (4)). Correspondingly, once the two spheres come into contact a finite normal force is suddenly induced due to auto-adhesion. Then the normal force increases with increasing the relative displacement until the relative velocity of the two spheres reaches zero, which corresponds to a maximum contact force. The normal force then starts to reduce until it reaches $-P_{C}$ as described by Eq. (5). Thereafter it reduces to zero at the moment of separation. The relative velocity increases first due to the autoadhesion and then decreases until a minimum value is reached, 
where the force reduces to zero. Thereafter it increases again until the two spheres separate from each other. On the other hand, without autoadhesion, both the relative displacement and the normal force firstly increase from zero to maximum values at which the velocity reaches zero, and then decrease to zero with time.

Fig. 4 shows the variation of the normalized contact force with the normalized relative displacement. The force acts on the two particles only when they are in contact with each other during the loading process, and the force increases with increasing displacement until a maximum value is reached. Then the unloading process starts and the force decreases as the displacement decreases. It is worth noting that the two particles do not separate from each other when the displacement reduces to zero due to the adhesion and they will continue moving in the opposite directions until the displacement reaches $\alpha_{F}$, at which point they separate from each other and the impact process ends. In Fig. 4, the theoretical prediction using JKR theory (i.e. Eq.(3)) is also superimposed. It is clear that the numerical results are in excellent agreement with the theoretical predictions, which implies that the model is correctly implemented and can be used to model impact behaviour of particles in the presence of adhesion.

When the initial relative velocity of the two particles is reduced to \pm 0.0050 $\mathrm{m} / \mathrm{s}$, the corresponding results are shown in Fig. 5 and Fig. 6. It can be seen from Fig. 5 that the loading and unloading processes are similar to those shown in Fig. 3, however, the two spheres cannot separate from each other when the unloading process ends. As a result, cyclic loading and unloading take place, and the relative displacement increases again, and the evolutions of normal force and velocity also repeat the similar trends as those in the first loading process. It is worth noting that the amplitudes of relative displacement, normal force and velocity monotonically decrease in the subsequent impacts and eventually the two spheres adhere to each other, since some kinetic energy is dissipated due to the adhesion in each impact. Fig. 6 shows the normalized impact force as a function of the normalized displacement. It can be seen that the loading and unloading curves are similar to those shown in Fig. 4. 
However, the two particles cannot separate from each other and will continue reloading until they stick together.

From the impacts with different initial velocities, it is found that due to the effect of the attractive force only the particles with sufficient initial kinetic energy (i.e. initial velocity) can rebound, while particles without sufficient initial kinetic energy will stick to each other. Therefore, there must be a critical initial velocity above which particles will rebound. An accurate determination of this critical velocity is crucial for the analysis of the attachment and detachment processes in DPIs and will be discussed in the next subsection.

\subsection{Critical Velocity}

As shown in Fig. 3, there is still a finite force acting on the spheres when the relative displacement reduces to zero during the unloading process. Thereafter the two spheres move in the opposite directions and the work done by the adhesive force dissipates some kinetic energy and possibly all the kinetic energy, which prevent the spheres separating. Therefore, only particles with sufficiently high kinetic energy will be able to rebound by overcoming the adhesive work, while others will stick to each other.

In order to accurately obtain the critical velocity, it is essential to calculate the work done by the adhesive force. Thornton [31] integrated the JKR force-displacement curve (described as Eq. (3)) and obtained the initial kinetic energy or the work to break the adhesive contact $W_{S}$ :

$$
W_{S}=7.09\left(\frac{\Gamma^{5} R^{4}}{E^{2}}\right)^{1 / 3}
$$

Therefore, if energy loss due to elastic wave propagation is negligible, particles will rebound only when

$$
\frac{1}{2} m_{1} V_{1}^{2}+\frac{1}{2} m_{2} V_{2}^{2} \geq W_{S}
$$

where $m_{1}, m_{2}$ are the mass of the two particles and $V_{1}, V_{2}$ are the impact velocities of the two particles.

For the cases $m_{1}=m_{2}, V_{1}=-V_{2}$, Eq. (10) can be rewritten as 


$$
\frac{1}{2} m^{*} V^{2} \geq W_{S}
$$

where

$$
m^{*}=\frac{m_{1} m_{2}}{m_{1}+m_{2}}
$$

and the relative impact velocity, $V$, is defined as

$$
V=\left|V_{1}-V_{2}\right|
$$

Using Eq. (9), the critical velocity, $V_{C}$, can be obtained as

$$
V_{C}=\left(\frac{14.18}{m^{*}}\right)^{1 / 2}\left(\frac{\Gamma^{5} R^{4}}{E^{2}}\right)^{1 / 6}
$$

For the cases in which two spheres rebound from each other, denoting the rebound velocities of the two spheres as $V_{1 r}$ and $V_{2 r}$, the following equation should be satisfied:

$$
\frac{1}{2} m_{1} V_{1}^{2}+\frac{1}{2} m_{2} V_{2}^{2}-\frac{1}{2} m_{1} V_{1 r}^{2}-\frac{1}{2} m_{2} V_{2 r}^{2}=W_{S}
$$

From Eq. (13) and Eq. (14), we have

$$
\frac{1}{2} m^{*} V_{C}^{2}=W_{S}
$$

Since $m_{1}=m_{2}, V_{1}=-V_{2}$ and $V_{1 r}=-V_{2 r}$, substituting Eq. (16) into Eq. (15) yields:

$$
V_{C}=\left(V^{2}-V_{r}^{2}\right)^{1 / 2}
$$

where the relative rebounding velocity $V_{r}$ is defined as

$$
V_{r}=\left|V_{1 r}-V_{2 r}\right|
$$

In the current numerical analysis, a low impact velocity was initially set for the two particles at which they will stick to each other after impact. The velocity was then incrementally increased until the two particles separate from each other after the impact. $V$ and $V_{r}$, defined in Eq. (13) and Eq. (18) respectively, can be obtained from the case in which two spheres 
rebound from each other, then the critical velocity can be obtained using Eq. (17).

As the critical velocity is crucial for the understanding of the attachment and detachment process in DPIs, different cases with different particle sizes and work of adhesion are then simulated to investigate their effects on the critical velocity. Fig. 7 shows that for the same material (i.e. the same work of adhesion, $\Gamma=0.44 \mathrm{~J} / \mathrm{m}^{2}$ ), the critical velocity decreases with increasing particle radius. Fig. 8 illustrates the effect of the work of adhesion on the critical velocity. For a given particle radius (i.e. $\left.R_{1}=R_{2}=100 \mu \mathrm{m}\right)$, the critical velocity increases with increasing work of adhesion. It can also be seen that the DEM results are in excellent agreement with the theoretical values obtained from Eq. (14), which indicates that the current model can accurately predict the critical velocity for impacts in the presence of adhesion.

\section{Mixing of a carrier with API particles in a vibrating container}

In this section, the mixing process of one carrier particle and a number of API particles in a vibrated container is analysed using DEM, and the effects of the vibration amplitude and frequency on the mixing performance are discussed.

\subsection{Vibration process}

Fig. 9 shows snapshots at various time instants during the vibration process for a typical case. It can be seen from Fig. 9a that a few API particles adhere to the carrier when the container starts to vibrate. During the vibration process the carrier and API particles oscillate in the vertical direction and an increasing number of impacts occurs between the carrier and API particles as shown in Fig. 9b. An increasing number of API particles stick to the carrier as the vibration continues (Fig. 9c).

Fig. 10 illustrates the corresponding evolution of the contact number $\left(N_{c}\right.$, the number of API particles contacting with the carrier). During the deposition process, a few API particles adhere to the carrier and a steady 
state value of $N_{c}$ is reached. Once the container starts to vibrate, the contact number increases as the time is increased until it reaches a stable status. Fluctuations are apparent during the vibration process due to the impact between the container walls and the carrier particle. The results in the stable state (i.e. time period in the rectangular box shown in Fig. 10) are further analysed to obtain the mean and standard deviation of the contact number, which will be discussed in the following sections.

\subsection{Effect of vibration velocity amplitude}

The variation of the mean and standard deviation of the contact number with vibration velocity amplitude, during the period indicated in the rectangular box in Fig. 10, is shown in Fig. 11. The vibration velocity amplitudes $V_{0}$ in these cases are different while the vibration frequency is fixed at $f=60 \mathrm{~Hz}$. The contact number first increases and then decreases with increasing vibration velocity amplitude. As a result there is optimal amplitude at which the contact number is a maximum. Moreover there exists a threshold amplitude below which the container cannot agitate API particles. On the other hand, the contact number will decrease due to "over-vibration" when the amplitude is larger than the optimal value.

The incremental contact number $\Delta N_{c}$ during the whole vibration process is also shown in Fig. 11. Impacts between the API particles and carrier particle are monitored during the process, then $\Delta N_{c}$ can be obtained by:

$$
\Delta N_{c}=N_{i} \eta_{s}-N_{d}
$$

where $N_{i}$ is the total impact number during the process, $\eta_{s}$ is the sticking efficiency that indicates the percentage of API particle adhering to the carrier after an impact, and $N_{d}$ is the total detachment number (the number of API particles which originally adhered to the carrier but are detached due to impact between the carrier particle and container wall or other removal forces). The variations of the impact number, sticking efficiency and detachment number as a function of the vibration amplitude are shown in Fig. 12. It can be seen that the impact number and detachment number both increase with increasing vibration amplitude 
while the sticking efficiency slightly decreases as the amplitude is increased. According to Eq. (19), the incremental contact number first increases and then decreases with increasing vibration amplitude. It is worth noting that the incremental contact number is obtained for the whole vibration process while the contact number gives the average value during the stable period highlighted in Fig. 10.

\subsection{Effect of vibration frequency}

Fig. 13 indicates the effect of vibration frequency on the contact number for a fixed vibration velocity amplitude $V_{0}=0.04 \mathrm{~m} / \mathrm{s}$. It shows that an optimal vibration frequency can be obtained as the contact number first increases and then decreases with an increase in the vibration frequency. Moreover, vibration with a very low frequency $(<40 \mathrm{~Hz})$ cannot agitate the particles efficiently as the contact number hardly increases during the vibration process. It also can be seen that the incremental contact number obtained by Eq. (19) presents a similar trend. Fig. 14 shows the variations of the impact number, sticking efficiency and detachment number as a function of the vibration frequency. The impact number and detachment number both increase with increasing vibration frequency while the sticking efficiency slightly decreases as the frequency is increased.

\section{Discussion}

\subsection{Critical velocity during impact between two spherical particles with adhesion}

A knowledge of the critical sticking velocity is very important for understanding the macroscopic behaviour of DPI formulations and provides science-based guidance for DPI formulation design. It is clear from the DEM simulations and predictions using Eq. (14) that increasing particle radius and decreasing work of adhesion can ideally decrease the critical velocity. This implies that, for impact-dominant dispersion processes, the dispersion performance in DPIs can be theoretically improved by using large particles with low work of adhesion, which will have a low critical sticking velocity so that particles can be dispersed more 
easily. It should be noted that the present analysis focuses on collinear impacts. Further analysis is needed for investigating the dispersion performance in processes dominated by oblique impacts of particles.

\subsection{Attachment process during mixing in a vibrating container}

Generally, the contact number during the attachment process is determined by the balance of the removal forces (i.e. impact force and gravitational force in the current cases) and adhesive forces (i.e. caused by the effect of the thermodynamic work of adhesion in the current cases) acting on the particles. However, the overall performance of attachment can be affected by many factors (e.g. vibration velocity amplitude and frequency). The current numerical analyses show that there is optimal vibration velocity amplitude and frequency that maximises the contact number. API particles are oscillated in the vertical direction within the vibrating container, which may impact with the carrier particle. Once the impact velocity is lower than the critical sticking velocity, API particles will theoretically stick to the carrier. The optimal amplitude and frequency corresponding to the maximum contact number should be sufficiently large to agitate API particles from the bottom of the container but sufficiently small in order to avoid too many particles that initially stick with the carrier but are detached during impacts when the vibrating velocity and frequency are too high (i.e., over-vibration). As shown in Fig. 12, the total impact number during the vibration process increases as the vibration velocity is increased, which indicates that API particles have more opportunities to impact with the carrier. On the other hand, as the vibration velocity is increased, the sticking efficiency decreases. This can be explained by the increasing impact velocity between the carrier and API particles with the increase of vibration velocity. As a result, many particles impact with the carrier with an impact velocity that is greater than the critical sticking velocity and thus rebound from the carrier. In addition, a higher vibration velocity causes more originally sticking API particles to detach from the carrier due to impacts between the carrier particle and the container walls. Therefore, there is an optimal vibration condition that maximises the contact number. 


\section{Conclusions}

A discrete element method taking into account the adhesion is employed to investigate the attachment processes in DPIs. The analysis of the impact behaviour of two elastic spheres shows that spheres with different initial kinetic energy can either stick or rebound. A critical sticking velocity from an energy analysis is introduced to determine whether two elastic spheres rebound or stick to each other during impact in the presence of adhesion. Moreover, it shows that the critical sticking velocity increases with increasing work of adhesion and decreasing particle radius. An excellent agreement between the numerical results and the theoretical predictions for the two-sphere impact indicates that DEM can be used to model impact behaviour of particles in the presence of adhesion. The attachment process of API particles with a carrier particle in a vibrating container is then investigated. It is shown that there is optimal vibration velocity amplitude and frequency to maximise the contact number. A close examination of the mixing process reveals that the impact number and detachment number during the vibration process both increase with increasing vibration amplitude or frequency while the sticking efficiency decreases as the amplitude or frequency is increased.

\section{Acknowledgements}

The first author would like to acknowledge the financial supports from the Chinese Scholarship Council (CSC) and the School of Chemical Engineering at the University of Birmingham through the Li Siguang scholarship scheme.

\section{Reference}

1. Islam, N., Gladki, E.: Dry powder inhalers (DPIs) - A review of device reliability and innovation. Int. J. Pharm. 360, 1-11 (2008)

2. Smith, I.J., Parry-Billings, M.: The inhalers of the future? A review of dry powder devices on the market today. Pulm. Pharmacol. Ther. 16, 79-95 (2003)

3. Newman, S.P., Busse, W.W.: Evolution of dry powder inhaler design, formulation, and performance. Respir. Med. 96, 293-304 (2002)

4. Pritchard, J.N.: The influence of lung deposition on clinical response. J. Aerosol med. 14, S19S26 (2001) 
5. Aulton, M.E., Taylor, K.: Pharmaceutics: The Science of Dosage Form Design. Churchill Livingstone, London (2001).

6. Hinds, W.C.: Aerosol Technology, Properties, Behavior, and Measurement of Airborne Particles, Wiley-Blackwell, New York (1999).

7. Kaialy, W., Alhalaweh, A., Velaga, S.P., Nokhodchi, A.: Influence of lactose carrier particle size on the aerosol performance of budesonide from a dry powder inhaler. Powder Technol. 227, 7485 (2012)

8. Guenette, E., Barrett, A., Kraus, D., Brody, R., Harding, L., Magee, G.: Understanding the effect of lactose particle size on the properties of DPI formulations using experimental design. Int. J. Pharm. 380, 80-88 (2009)

9. Hassan, M.S. Lau, R.: Inhalation performance of pollen-shape carrier in dry powder formulation with different drug mixing ratios: Comparison with lactose carrier. Int. J. Pharm. 386, 6-14 (2010) 10. Steckel, H. Bolzen, N.: Alternative sugars as potential carriers for dry powder inhalations. Int. J. Pharm. 270, 297-306 (2004)

11. Young, P.A., Edge, S., Traini, D., Jones, M.D., Price, R., El-Sabawi, D., Urry, C., Smith, C.: The influence of dose on the performance of dry powder inhalation systems. Int. J. Pharm. 296, 26-33 (2005)

12. Steckel, H. and Muller, B.W.: In vitro evaluation of dry powder inhalers .2. Influence of carrier particle size and concentration on in vitro deposition. Int. J. Pharm. 154, 31-37 (1997)

13. Kaialy, W., Alhalaweh, A., Velaga, S.P., and Nokhodchi,A.: Effect of carrier particle shape on dry powder inhaler performance. Int. J. Pharm. 421, 12-23 (2011)

14. Kaialy, W., Martin, G.P., Larhrib, H., Ticehurst, M.D., Kolosionek, E., Nokhodchi, A.: The influence of physical properties and morphology of crystallised lactose on delivery of salbutamol sulphate from dry powder inhalers. Colloids Surf. B-Biointerfaces 89, 29-39 (2012)

15. Kaialy, W., Ticehurst, M., Nokhodchi, A.: Dry powder inhalers: Mechanistic evaluation of lactose formulations containing salbutamol sulphate. Int. J. Pharm. 423, 184-194 (2012)

16. Young, P.M., Roberts, D., Chiou, H., Rae, W., Chan, H.K., Traini,D.: Composite carriers improve the aerosolisation efficiency of drugs for respiratory delivery. J. Aerosol Sci. 39, 82-93 (2008)

17. Le, V., Thi, T., Robins, E., Flament, M.: Dry Powder Inhalers: Study of the Parameters Influencing Adhesion and Dispersion of Fluticasone Propionate. AAPS PharmSciTech 13, 477-484 (2012)

18. Jashnani, R.N., Byron, P.R., Dalby, R.N.: Testing of Dry Powder Aerosol Formulations in Different Environmental-Conditions. Int. J. Pharm. 113, 123-130 (1995)

19. Cline, D., Dalby, R.: Predicting the Quality of Powders for Inhalation from Surface Energy and Area. Pharm. Res. 19, 1274-1277 (2002)

20. Kaialy, W., Nokhodchi, A.: Antisolvent crystallisation is a potential technique to prepare engineered lactose with promising aerosolisation properties: Effect of saturation degree. Int. J.

Pharm. 437, 57-69 (2012) 
21. Shur, J., Kubavat, H.A., Ruecroft, G., Hipkiss, D., Price, R.: Influence of crystal form of ipratropium bromide on micronisation and aerosolisation behaviour in dry powder inhaler formulations. J. Pharm. Pharmacol. 64, 1326-1336 (2012)

22. Selvam, P., Marek, S., Truman, C., McNair, D., Smyth, H.D.: Micronized Drug Adhesion and Detachment from Surfaces: Effect of Loading Conditions. Aerosol Sci. Technol. 45, 81-87 (2011) 23. Jones, M.D. and Price, R.: The influence of fine excipient particles on the performance of carrier-based dry powder inhalation formulations. Pharm. Res. 23, 1665-1674 (2006)

24. Zeng, X.M., Martin, G.P., Tee, S.K., Abu Ghoush, A., Marriott,C.: Effects of particle size and adding sequence of fine lactose on the deposition of salbutamol sulphate from a dry powder formulation. Int. J. Pharm. 182, 133-144 (1999)

25. Saleem, I., Smyth, H., Telko, M.: Prediction of dry powder inhaler formulation performance from surface energetics and blending dynamics. Drug Dev. Ind. Pharm. 34: 1002-1010 (2008) 26. Dickhoff, B.H.J., De Boer, A.H., Lambregts, D., Frijlink, H.W.: The effect of carrier surface and bulk properties on drug particle detachment from crystalline lactose carrier particles during inhalation, as function of carrier payload and mixing time. Eur. J. Pharm. Biopharm. 56, 291-302 (2003)

27. Thornton, C. Yin, K.K.: Impact of Elastic Spheres with and Without Adhesion. Powder Technol. 65, 153-166 (1991)

28. Johnson, K.L., Kendall, K., Roberts, A.D.: Surface Energy and the Contact of Elastic Solids. Proc. R. Soc. Lond. A 324, 301-313 (1971)

29. Johnson, K.L.: Contact Mechanics, Cambridge University Press, Cambridge (1985).

30. Alderborn, G. Nyström, C.: Pharmaceutical Powder Compaction Technology, Marcel Dekker, New York (1996)

31. Thornton, C. Ning, Z.M.: A theoretical model for the stick/bounce behaviour of adhesive, elastic-plastic spheres. Powder Technol. 99, 154-162 (1998) 
Table 1 Physical properties of particles for the impact between two spheres

\begin{tabular}{ll}
\hline Parameter & Value \\
\hline Diameter $(\mu \mathrm{m})$ & $1-100$ \\
Density $\left(\mathrm{kg} / \mathrm{m}^{3}\right)$ & 2650 \\
Young's modulus $(\mathrm{GPa})$ & 70 \\
Poisson's ratio & 0.3 \\
Coefficient of friction & 0.35 \\
Thermodynamic work of adhesion $\left(\mathrm{J} / \mathrm{m}^{2}\right)$ & $0.044-4.4$ \\
\hline
\end{tabular}


Table 2 Simulation parameters for mixing of the carrier with APIs in a vibrating container

\begin{tabular}{ll}
\hline Parameter & Value \\
\hline Density $\left(\mathrm{kg} / \mathrm{m}^{3}\right)$ & 2650 \\
Young's modulus $(\mathrm{GPa})$ & 24 \\
Poisson's ratio & 0.3 \\
Friction coefficient & 0.3 \\
Thermodynamic work of adhesion $\left(\mathrm{J} / \mathrm{m}^{2}\right)$ & 0.006 \\
$V_{0}(\mathrm{~m} / \mathrm{s})$ & $0.02-0.06$ \\
$f(\mathrm{~Hz})$ & $30-120$ \\
$l(\mu \mathrm{m})$ & 105 \\
$N$ & 200 \\
\hline
\end{tabular}




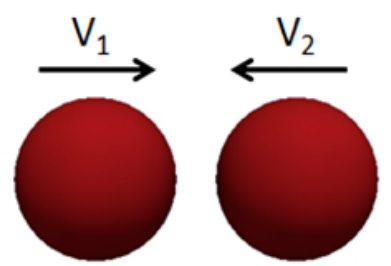

Fig. 1 Illustration of the normal impact between two spheres 


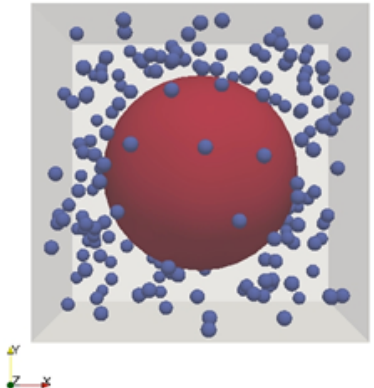

a)

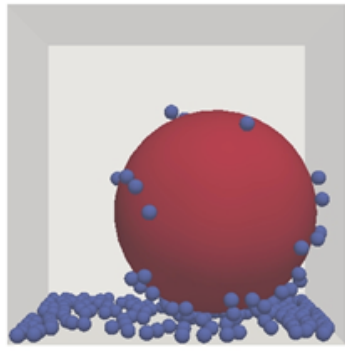

b)

Fig. 2 Model setup for vibrating container cases 

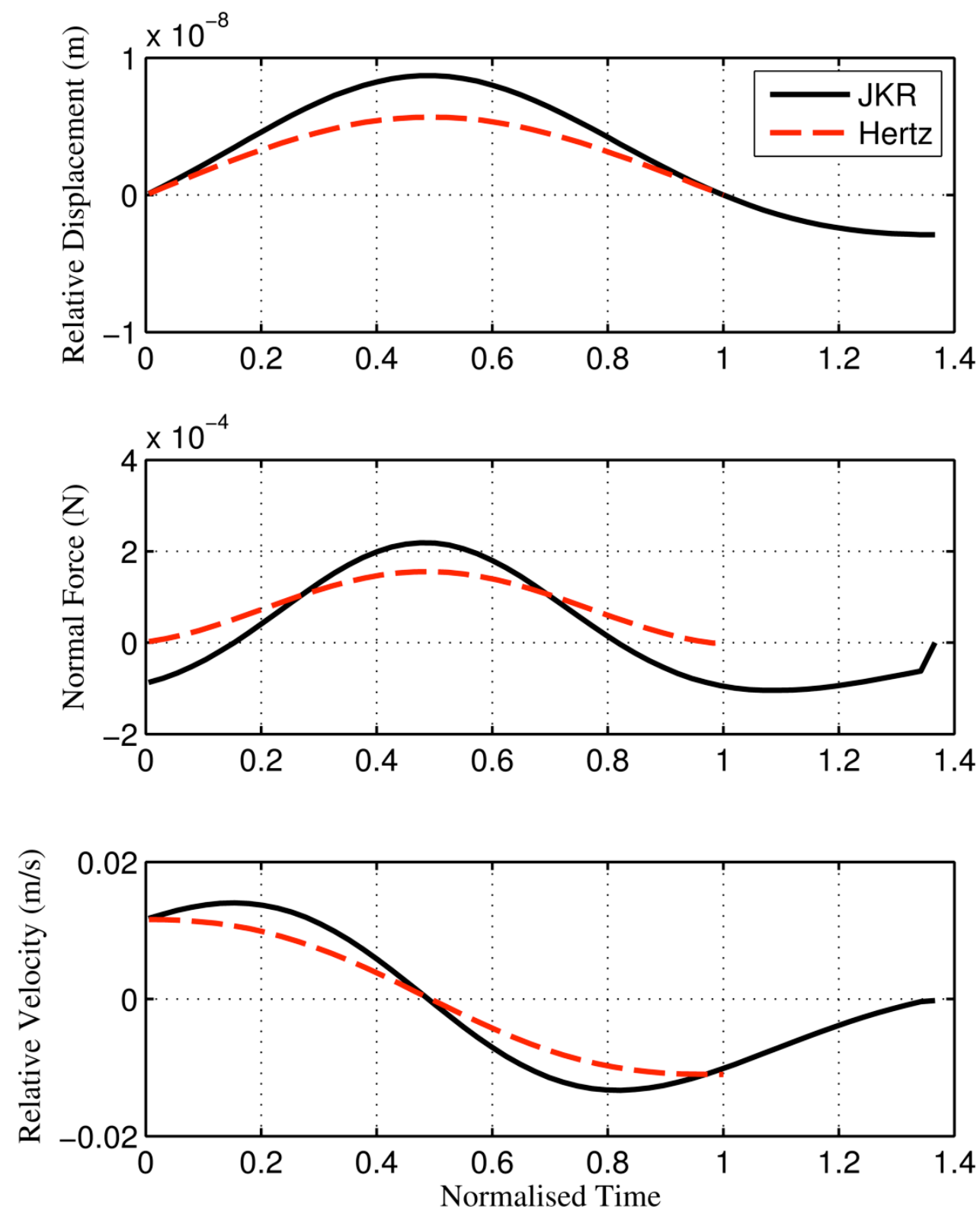

Fig. 3 The evolution of the relative displacement, relative velocity and normal force for the impact between two particles with an initial velocity of $\pm 0.0058 \mathrm{~m} / \mathrm{s}$ 


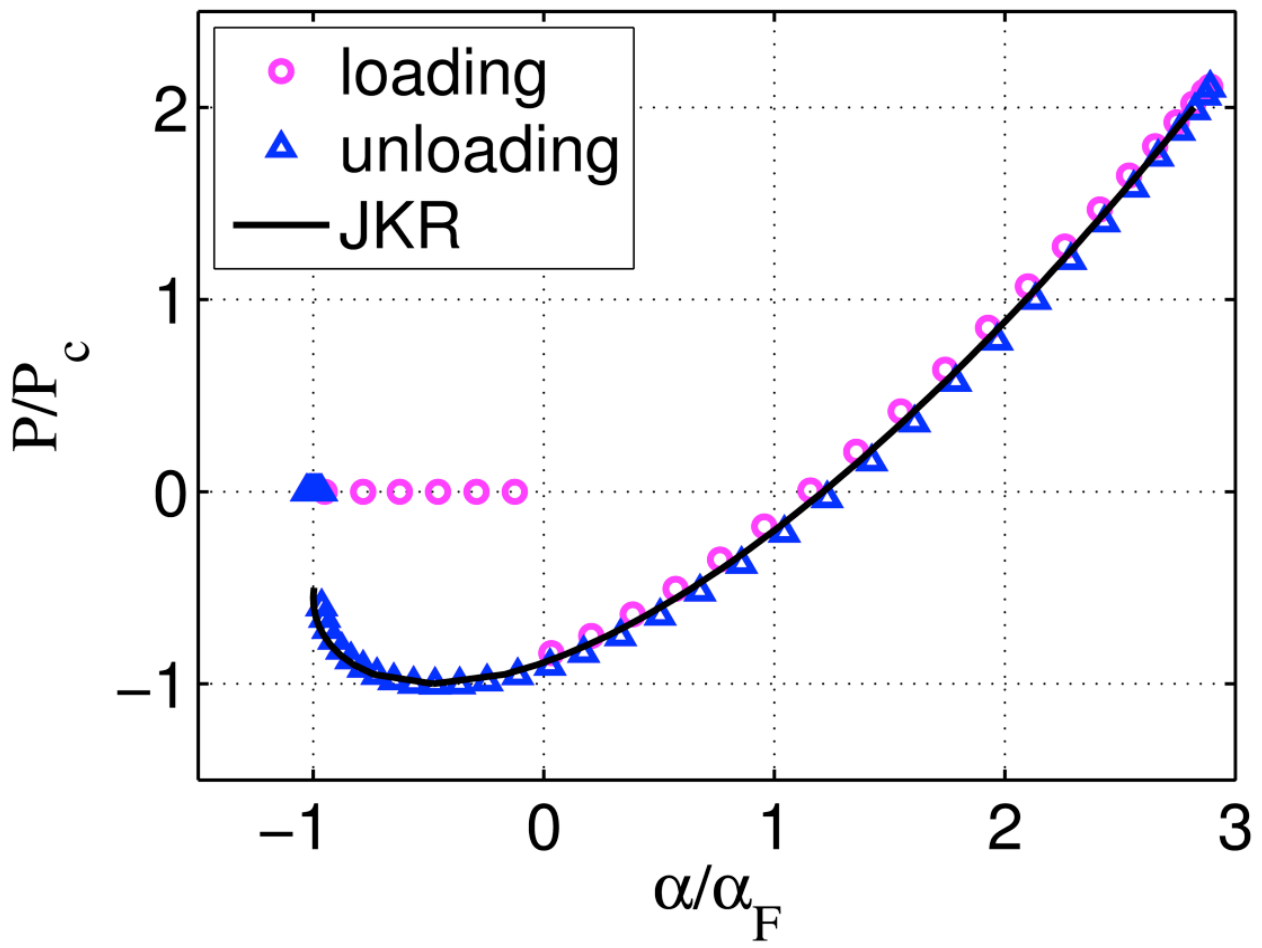

Fig. 4 The force-displacement relationship for impact between two particles with an initial velocity of $\pm 0.0058 \mathrm{~m} / \mathrm{s}$ 

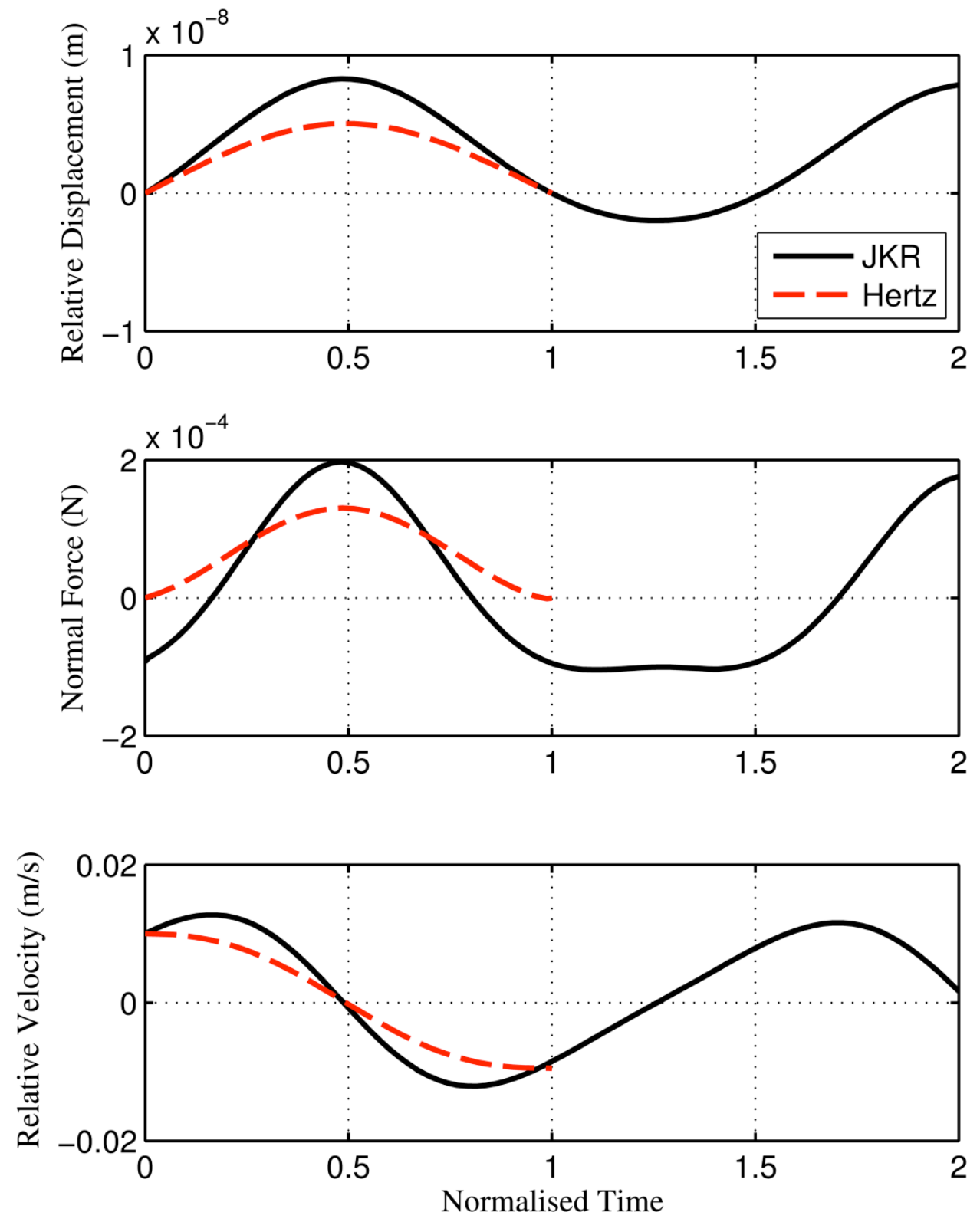

Fig. 5 The evolution of relative displacement, relative velocity and normal force for the impact between two particles with an initial velocity of $\pm 0.0050 \mathrm{~m} / \mathrm{s}$ 


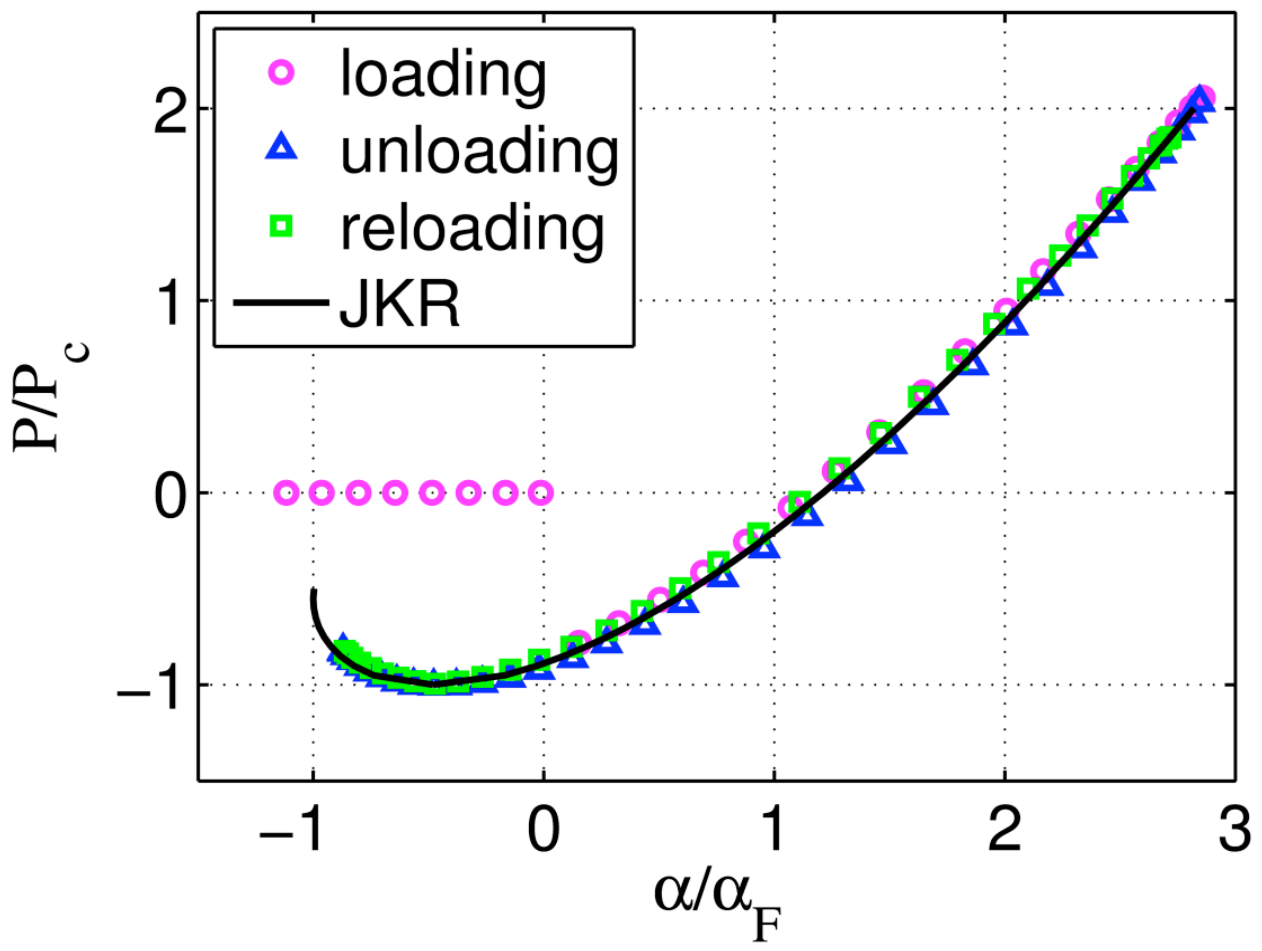

Fig. 6 The force-displacement relationship for the impact between two particles with an initial velocity of $\pm 0.0050 \mathrm{~m} / \mathrm{s}$ 


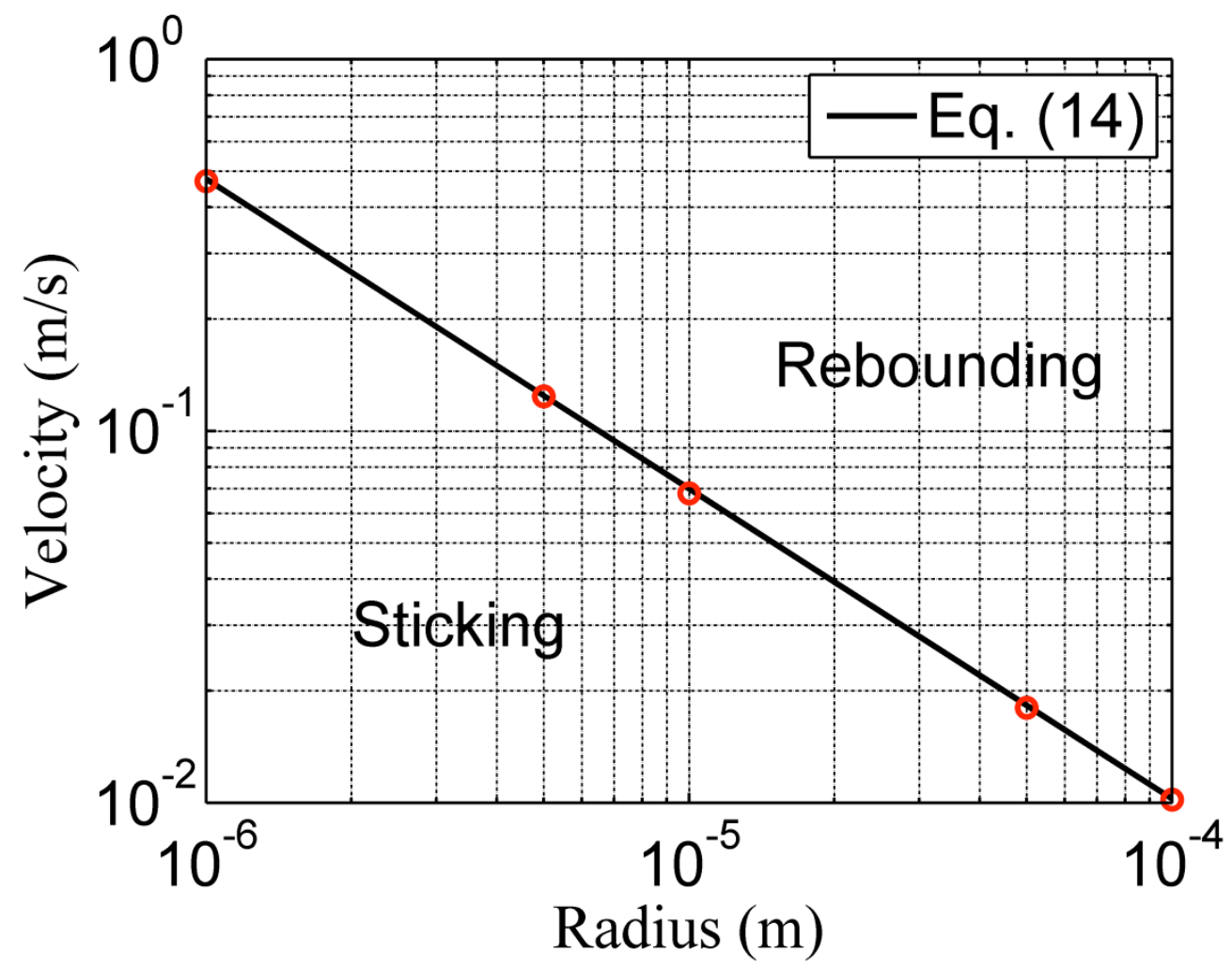

Fig. 7 The variation of the critical sticking velocity with particle radius ( $\Gamma=0.44 \mathrm{~J} / \mathrm{m}$ ) 


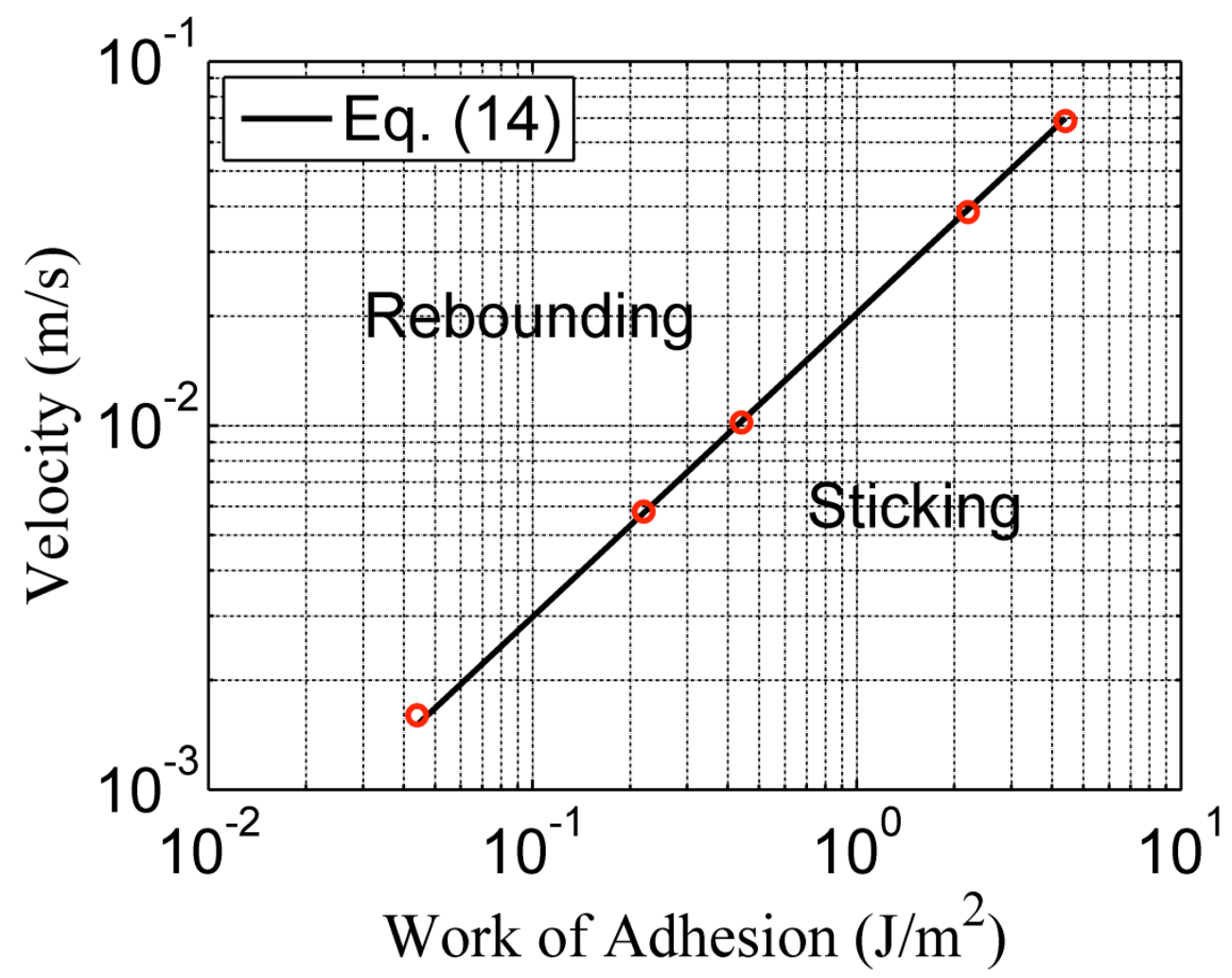

Fig. 8 The variation of the critical sticking velocity with the work of adhesion $\left(R_{1}=R_{2}=100 \mu \mathrm{m}\right)$ 


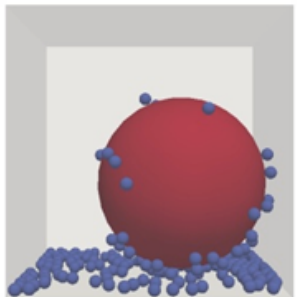

a) $\mathrm{t}=0.0750 \mathrm{~s}$

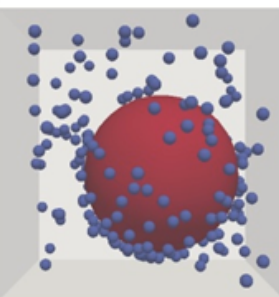

b) $t=0.1205 \mathrm{~s}$

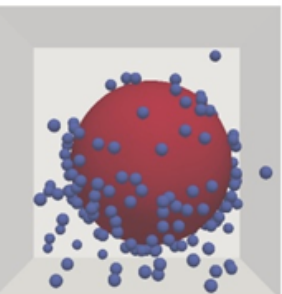

c) $t=0.2251 \mathrm{~s}$

Fig. 9 Snapshots at different time instances 


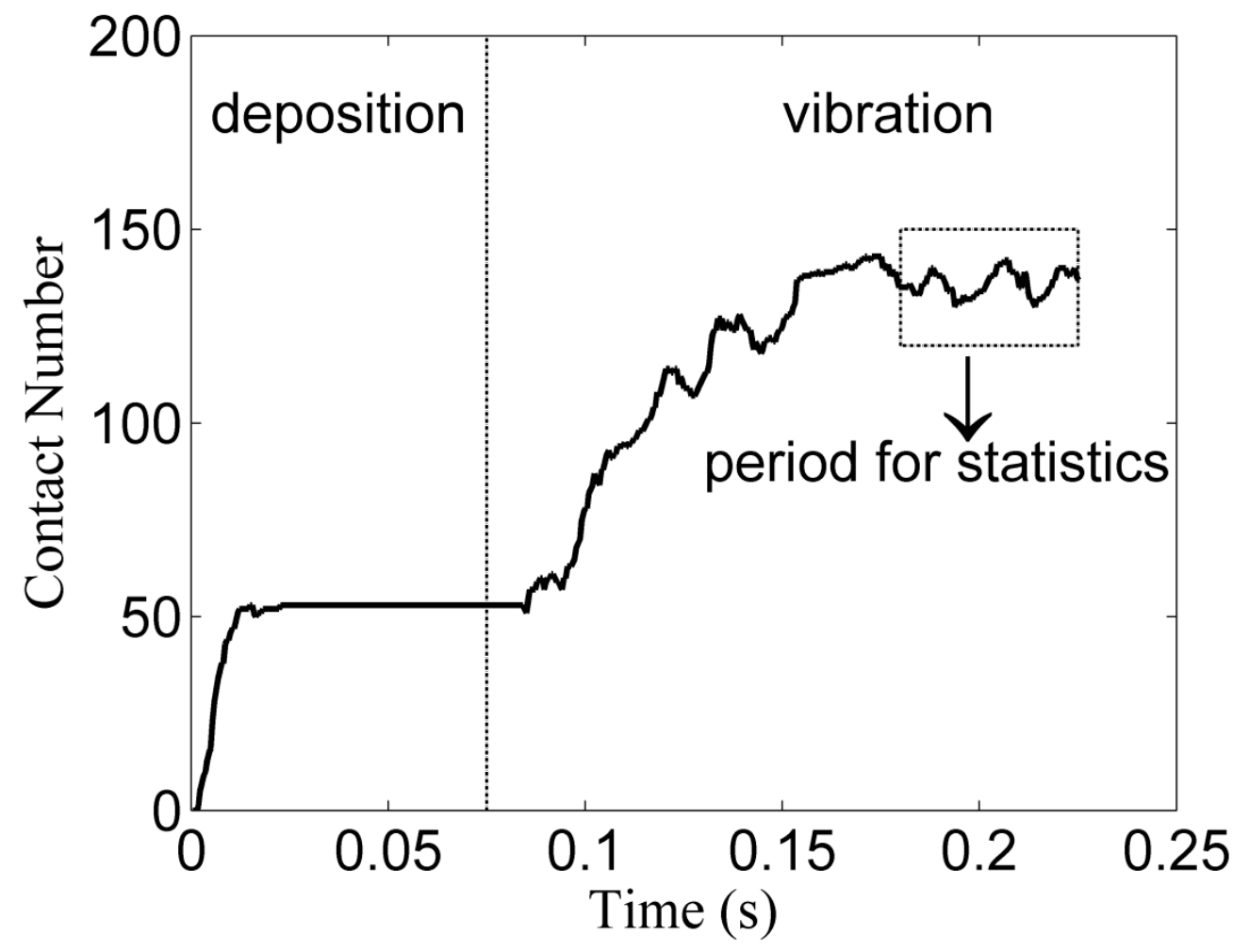

Fig. 10 The evolution of the contact time 


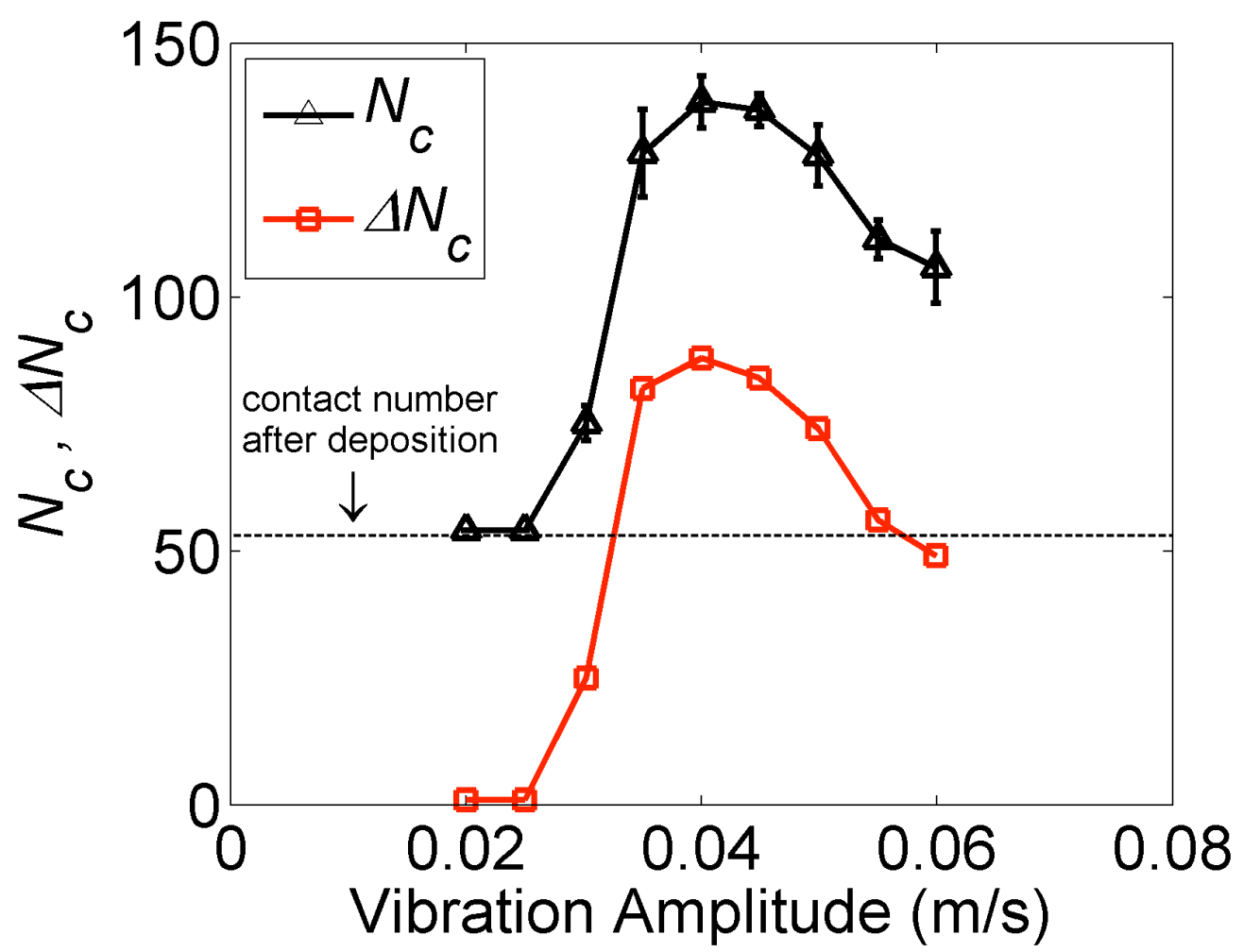

Fig. 11 The variation of contact number with the vibration velocity amplitude 

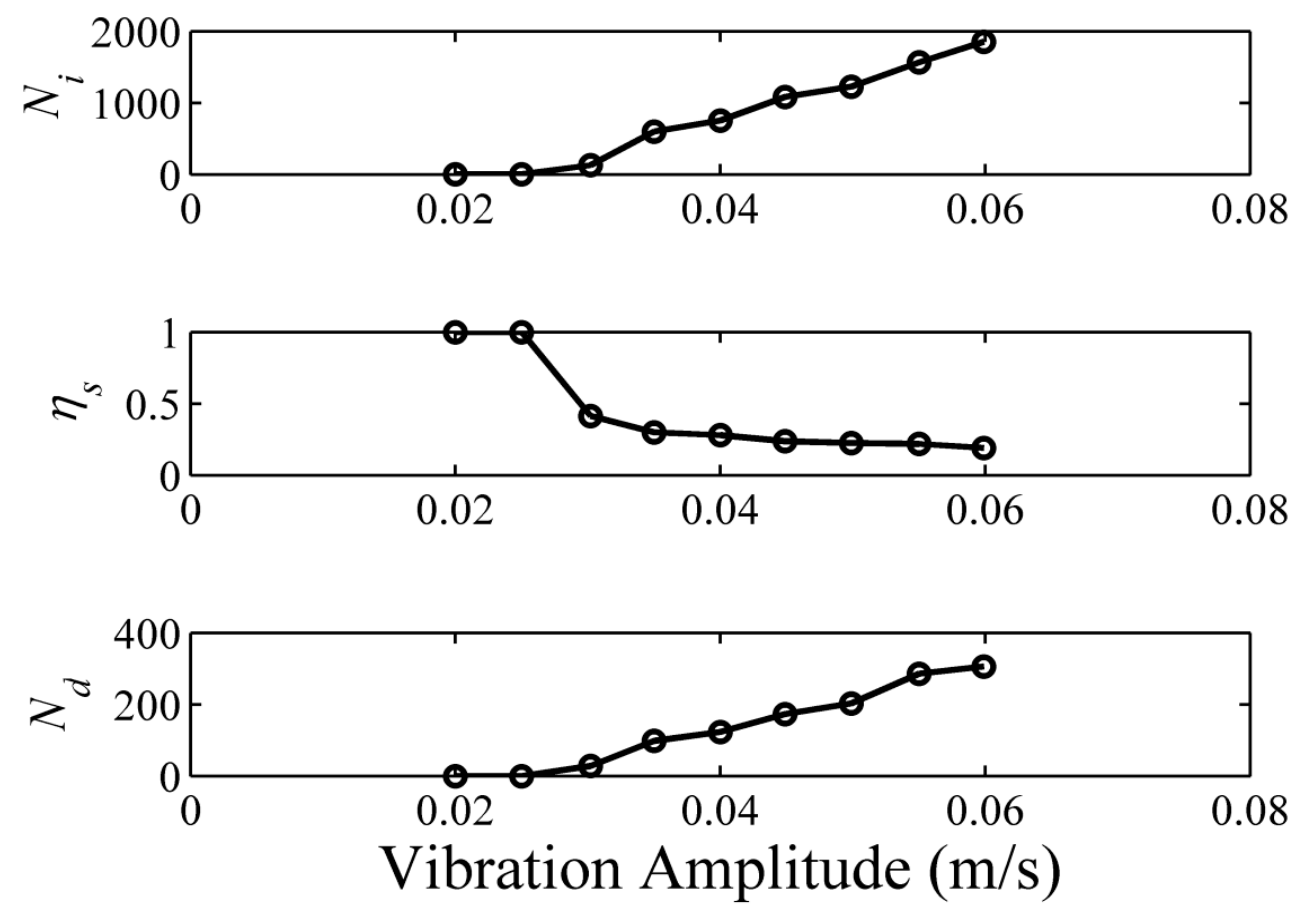

Fig. 12 The variations of the impact number, sticking efficiency and detachment number as a function of vibration amplitude 


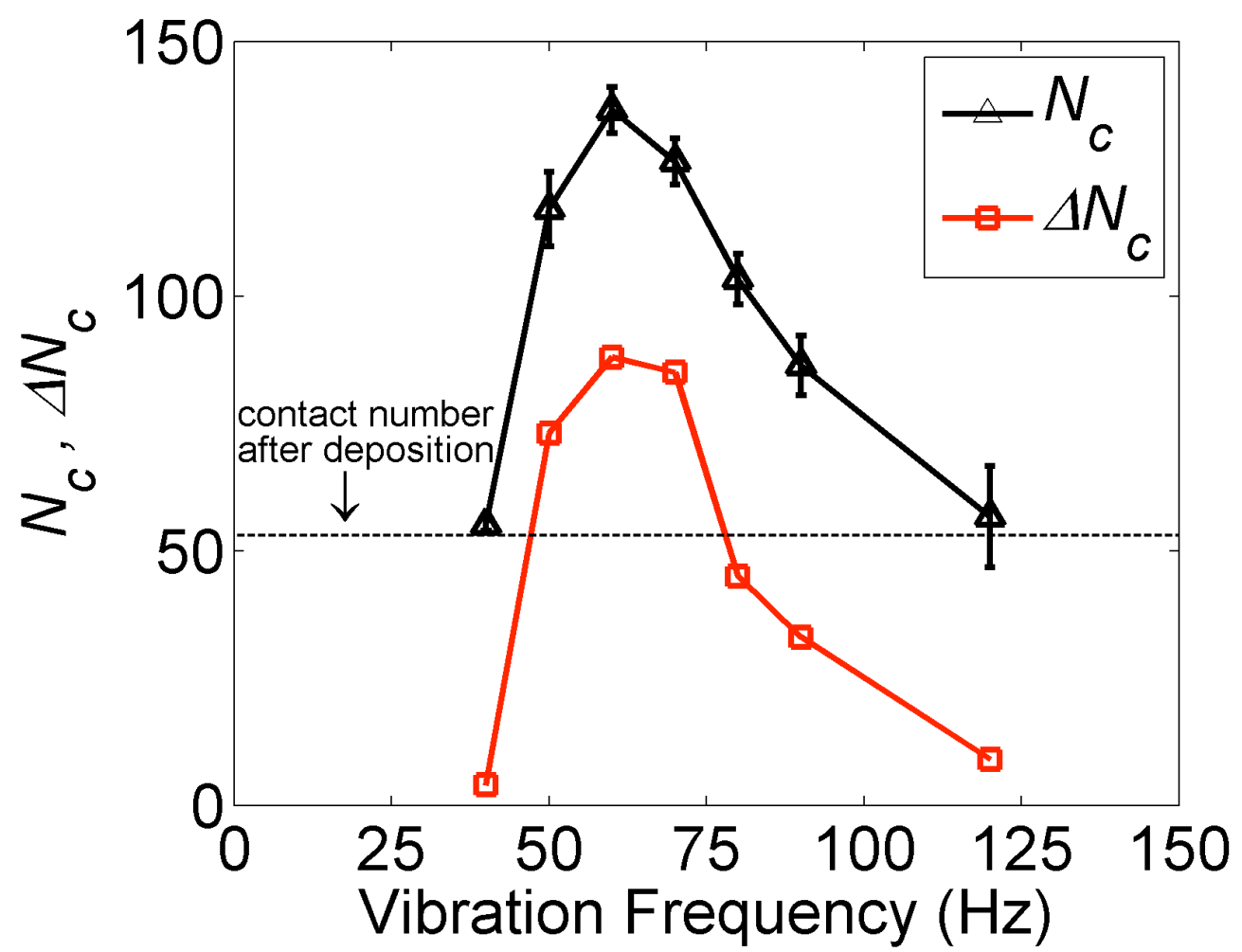

Fig. 13 The variation of contact number with the vibration frequency 

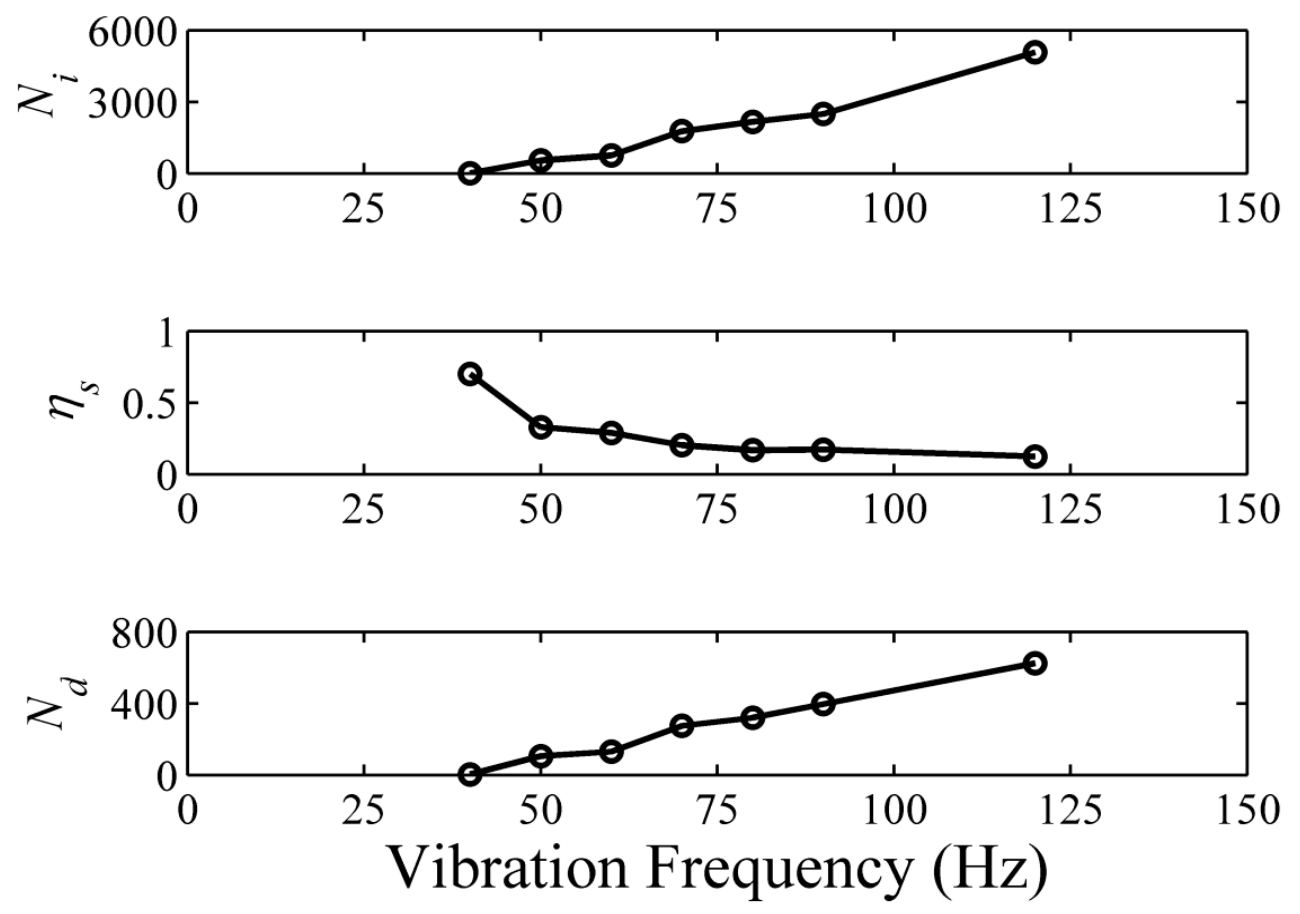

Fig. 14 The variations of impact number, sticking efficiency and detachment number against vibration frequency 